\title{
Disphenoidal Zero-Dimensional Lead, Tin, and Germanium Halides: Highly Emissive Singlet and Triplet Self-Trapped Excitons and X-ray Scintillation
}

\author{
Viktoriia Morad, ${ }^{\dagger, \ddagger}$ Yevhen Shynkarenko, ${ }^{\dagger, \ddagger}$ Sergii Yakunin, ${ }^{\dagger, \ddagger(0)}$ Alexandra Brumberg, ${ }^{\S}$ \\ Richard D. Schaller, ${ }^{\S} \|_{\odot}$ and Maksym V. Kovalenko ${ }^{* \dagger, \ddagger(0)}$ \\ ${ }^{\dagger}$ Laboratory of Inorganic Chemistry, Department of Chemistry and Applied Bioscience, ETH Zürich, Vladimir Prelog Weg 1, \\ CH-8093 Zürich, Switzerland \\ ${ }^{\ddagger}$ Laboratory for Thin Films and Photovoltaics, Empa-Swiss Federal Laboratories for Materials Science and Technology, \\ Überlandstrasse 129, CH-8600 Dübendorf, Switzerland \\ ${ }^{\S}$ Department of Chemistry, Northwestern University, 2145 Sheridan Road, Evanston, Illinois 60208, United States \\ "Center for Nanoscale Materials, Argonne National Laboratory, 9700 Cass Avenue, Lemont, Illinois 60439, United States
}

Supporting Information

\begin{abstract}
Low-dimensional metal halides have been researched as optoelectronic materials for the past two decades. Zero-dimensional halides of $\mathrm{ns}^{2}$ elements ( $\mathrm{Sn}$, $\mathrm{Pb}, \mathrm{Sb}$ ) have recently gained attention as highly efficient broadband light emitters. These compounds comprise discrete metal halide centers, isolated by bulky organic cations. Herein, we report isostructural halide complexes of $\mathrm{Ge}(\mathrm{II}), \mathrm{Sn}(\mathrm{II})$, and $\mathrm{Pb}$ (II) with a 1-butyl-1-methylpiperidinium cation (Bmpip), featuring unusual disphenoidal coordination with a highly stereoactive lone pair. Spectrally broad, bright emission from highly localized excitons, with quantum efficiencies of up to $75 \%$, is observed in blue to red spectral regions for bromides (for $\mathrm{Pb}, \mathrm{Sn}$, and $\mathrm{Ge}$, respectively) and extends into the nearinfrared for Bmpip $_{2} \mathrm{SnI}_{4}$ (peak at $730 \mathrm{~nm}$ ). In the case of $\mathrm{Sn}$ (II) and $\mathrm{Ge}(\mathrm{II})$, both singlet and triplet excitonic emission bands have been observed. Furthermore, $\mathrm{Bmpip}_{2} \mathrm{SnBr}_{4}$ and $\mathrm{Bmpip}_{2} \mathrm{PbBr}_{4}$ exhibit X-ray-excited luminescence (radioluminescence) with brightness being commensurate with that of a commercial inorganic X-ray scintillator (NaI:Tl).
\end{abstract}

$\mathrm{D}$ iverse metal halides are increasingly recognized for their potential as versatile classical light sources (lighting, displays) ${ }^{1}$ and quantum light emitters (single-photon sources, entangled photon). ${ }^{2}$ Recent years had seen a surge of reports on lead and tin halide perovskites, whose atomistic structure comprises corner-sharing metal halide octahedra, leading to a delocalized electronic structure with large Wannier-type excitons characterized by small binding energies (10-40 $\mathrm{meV}$ ): the attributes most suited for light harvesting purposes (solar cells, photodetectors). ${ }^{3}$ For a related but rather orthogonal purpose-enhancing the light emissivity-engineering the electronic dimensionality for controlling the formation and radiative recombination of the excitons had been a major thrust. This can be accomplished either morphologically, that is, by tuning the crystal size in one or more dimensions (e.g., nanocrystals), ${ }^{4}$ or structurally, i.e., by controlling the connectivity of metal halide polyhedral anions. ${ }^{5}$ As the structural dimensionality decreases, so also does the electronic structure, favoring the formation of the smaller, strongly bound excitons already at room temperature (RT), starting from 2-dimensional (2D) compounds. ${ }^{6}$ Electronic localization culminates in OD metal halides: ${ }^{7}$ a photoexcited electron-hole pair resides within one anionic entity, and its formation and subsequent relaxation can be described as electronic transitions in the central metal atom, ${ }^{8}$ perturbed by the field of halide ligands. Luminescence from $0 D$ metal halides is typically characterized by large Stokes shifts and broad emission line widths: both features in agreement with the strong coupling of the excitons to multiple phonons (e.g., structural distortions of the metal halide anions).

Herein, we report on highly luminescent $0 \mathrm{D}$ hybrid organic-inorganic germanium(II), tin(II), and lead(II) bromides and tin(II) iodide, comprising $\mathrm{MX}_{4}{ }^{2-}$ anions of a rare disphenoidal geometry surrounded by 1-butyl-1-methylpiperidinium (Bmpip) cations. Having homologue series of isostructural $\mathrm{Bmpip}_{2} \mathrm{MBr}_{4}$ allowed for assessment of the effect of the central metal, in particular, stereoactivity of its lone pair. These compounds also exhibit pronounced singlet exciton emission at RT, in addition to triplet emission (also known as self-trapped excitons). All presented compounds are bright broadband emitters in the visible spectral region, with photoluminescence quantum yields (PL QYs) of up to $75 \%$. $\mathrm{PL}$ of $\mathrm{Bmpip}_{2} \mathrm{SnI}_{4}$ extends into the near-infrared region.

$\mathrm{Bmpip}_{2} \mathrm{SnBr}_{4}$ and $\mathrm{Bmpip}_{2} \mathrm{PbBr}_{4}$ also exhibit intense emission under X-ray excitation, motivating further work on the exploration of low-dimensional metal halides for X-ray scintillation.

The electronic spectra of $\mathrm{ns}^{2}$ ions in solid matrixes and solutions have been widely investigated over the past century. Singlet and triplet bound excitons, as well as emission from defects, were observed at low temperatures in, for instance,

Received: March 16, 2019

Published: June 17, 2019 

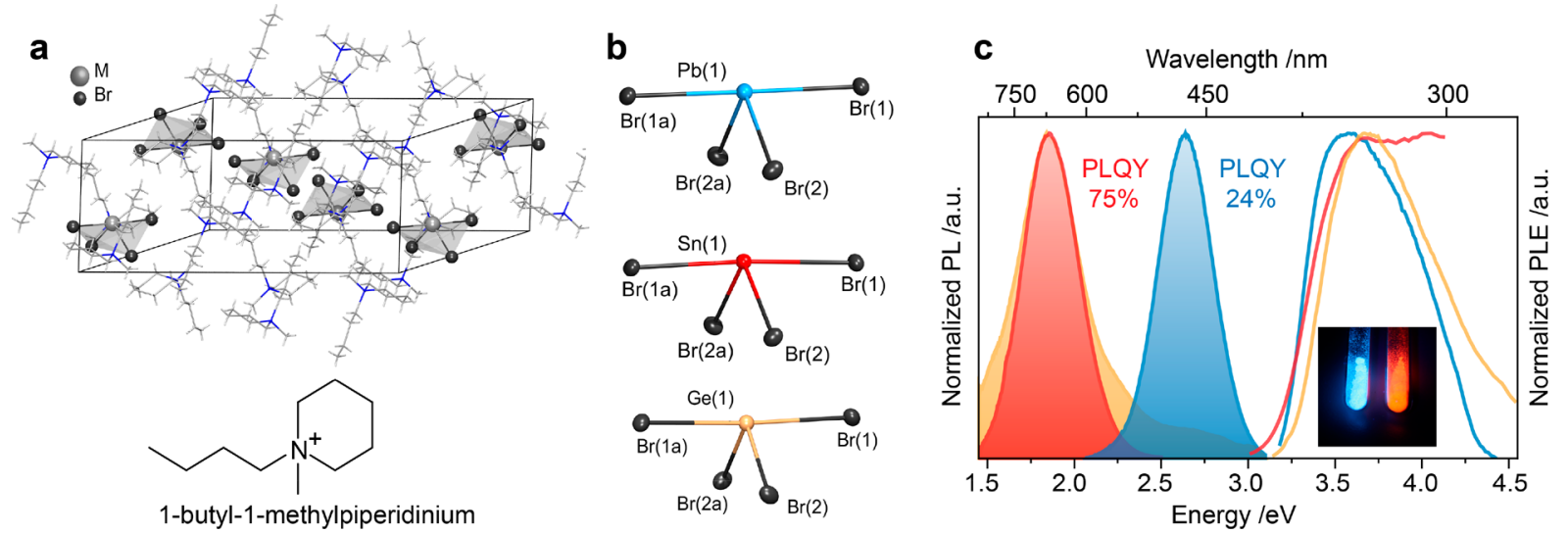

Figure 1. (a) Crystal structure of $\mathrm{Bmpip}_{2} \mathrm{MBr}_{4}$, viewed along the [110] crystallographic direction. (b) Disphenoidal coordination of lead, tin, and germanium, demonstrating a different degree of repulsion between axial bromide atoms and the lone pair, which occupies one of the equatorial positions. (c) RT spectra of PL (color-shaded) and PLE for Bmpip $\mathrm{PbBr}_{4}$ (blue), Bmpip $\mathrm{SnBr}_{4}$ (red), and $\mathrm{Bmpip}_{2} \mathrm{GeBr}_{4}$ (yellow) and (inset) a photograph of $\mathrm{Bmpip}_{2} \mathrm{PbBr}_{4}$ and $\mathrm{Bmpip}_{2} \mathrm{SnBr}_{4}$ under UV excitation.

$\mathrm{PbCl}_{2}, \mathrm{PbBr}_{2}, \mathrm{PbF}_{2}$, and $\mathrm{SnCl}_{2} .{ }^{9}$ Such $\mathrm{ns}^{2}$ metals possess a lone pair that can be either inert or stereochemically active, depending on the efficiency of s- and p-orbital mixing. The mixing of states for the $\mathrm{Pb}$ lone pair is recognized to give rise to important phenomena in lead halide perovskites, including defect tolerance. ${ }^{10}$ However, the toxicity of lead motivates the quest for benign alternatives such as its electronic analoguestin and germanium.

In general, organic-inorganic metal halide hybrids achieve greater structural versatility due to the structural engineerabilty of the organic moiety: its bulkiness and symmetry dictate the anionic framework of the structure. Bmpip is an example of a bulky cation with weak intermolecular interactions (lack of hydrogen-bonding or $\pi$-stacking capabilities), explaining the low melting points of the obtained compounds (Table S1). $\mathrm{Bmpip}_{2} \mathrm{PbBr}_{4}, \mathrm{Bmpip}_{2} \mathrm{SnBr}_{4}, \mathrm{Bmpip}_{2} \mathrm{GeBr}_{4}$, and $\mathrm{Bmpip}_{2} \mathrm{SnI}_{4}$ were synthesized from precursor solutions of metal(II) halide and BmpipX in organic solvents and have been structurally studied by single-crystal and powder X-ray diffraction (Figure $\mathrm{S} 1$; space group, $\mathrm{C} 2 / c$; see Tables $\mathrm{S} 2-\mathrm{S} 5$ for further details). Figure 1a illustrates the unit cell. The coordination of lead(II) with $C_{2 v}$ symmetry of the $\mathrm{ns}^{2}$ ion had been previously described in the systems with bulky phosphonium salts. ${ }^{11}$ It results from the deviation from $T_{d}$ tetrahedral symmetry due to HOMO stabilization. For the tin(II) bromide anion, a similar coordination has been recently reported. ${ }^{12}$

All three complexes exhibit broadband luminescence at room temperature (RT) upon excitation with UV light (Figure 1c, Table S14 and Figure S2). While the excitation bands of all compounds are peaked at similar energies (ca. $3.5 \mathrm{eV}$ ), the PL band of $\mathrm{Bmpip}_{2} \mathrm{PbBr}_{4}$ centers in the blue $(470 \mathrm{~nm})$, and that of germanium or tin counterparts shifts to red (peaks at $660-670$ $\mathrm{nm}$ ). The emission from the germanium complex is broader, likely due to stronger vibronic coupling. $\mathrm{Bmpip}_{2} \mathrm{SnI}_{4}$ is further shifted to the near-infrared (peak at $730 \mathrm{~nm}$, Figure S3). $\mathrm{Bmpip}_{2} \mathrm{SnBr}_{4}$ exhibits the highest PL QY of $75 \%, \mathrm{Bmpip}_{2} \mathrm{PbBr}_{4}$ $24 \%$, and $\mathrm{Bmpip}_{2} \mathrm{GeBr}_{4}$ display the lowest PL QY below $1 \%$ (Table S14).

Fundamentally important is the observation of both singlet and triplet emission bands in all $\mathrm{Bmpip}_{2} \mathrm{MBr}_{4}$, wellpronounced for $\mathrm{Sn}$ and $\mathrm{Ge}$ at $\mathrm{RT}$, when sufficiently highenergy photons are used for excitation (above $3.9 \mathrm{eV}$, Figure $2 \mathrm{a}-\mathrm{c}$, Figure S4). The blue emission band (singlet) can be
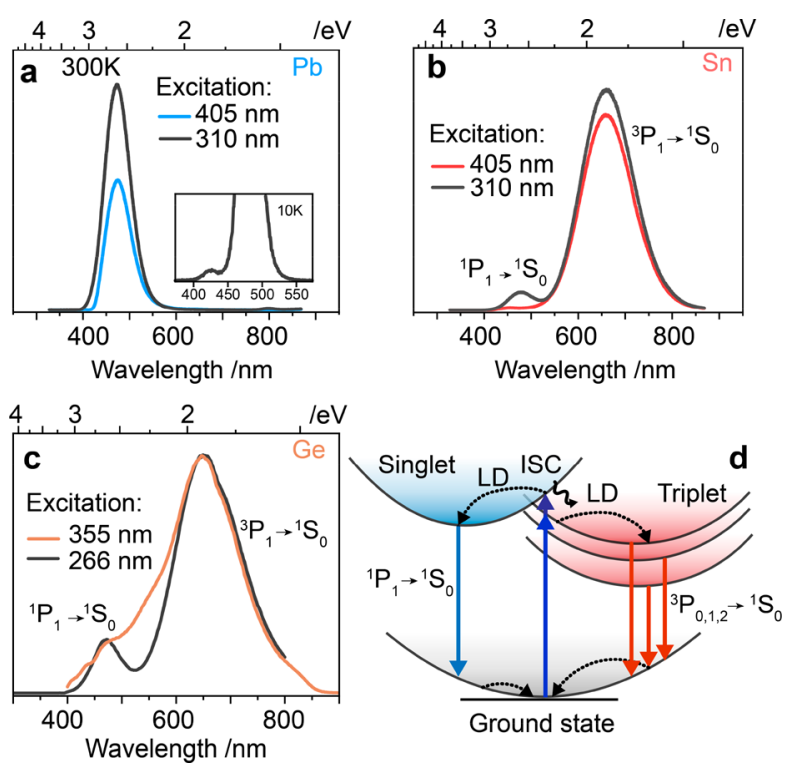

Figure 2. $(\mathrm{a}-\mathrm{c}) \mathrm{PL}$ spectra of $\mathrm{Bmpip}_{2} \mathrm{MBr}_{4}$ at different excitation wavelengths, showing both singlet and triplet emission bands for Sn, $\mathrm{Ge}(\mathrm{RT})$, and $\mathrm{Pb}$ (inset, $10 \mathrm{~K}$ ) cases. (d) Single coordinate diagram demonstrating the accessibility of both singlet and triplet bound excitonic states in $\mathrm{Bmpip}_{2} \mathrm{MBr}_{4}$. LD, lattice distortion; ISC, intersystem crossing.

attributed to the ${ }^{1} \mathrm{P}_{1} \rightarrow{ }^{1} \mathrm{~S}_{0}$ transition, whereas the lowerenergy emission band can be attributed to the ${ }^{3} \mathrm{P}_{n} \rightarrow{ }^{1} \mathrm{~S}_{0}$ transitions (where $n$ can be $0,1,2$ ) (Scheme S1). For example, in $\mathrm{Bmpip}_{2} \mathrm{SnBr}_{4}$, this assignment is corroborated by emission decay times (Figure S5) of $\tau=10 \mathrm{~ns}$ (singlet) and $\tau=4 \mu \mathrm{s}$ (triplet). Each singlet or triplet band in $\mathrm{Bmpip}_{2} \mathrm{GeBr}_{4}$ consists of two components (Figure S5). Cooling from 300 to $150 \mathrm{~K}$ resulted in the disappearance of the shorter component (Figures S6a and S7, streak-camera experiments). This could be explained by the shorter component of the decay originating from the efficient exciton coupling to a phonon mode activated above $150 \mathrm{~K}$. For $\mathrm{Bmpip}_{2} \mathrm{SnBr}_{4}$, no such pronounced temperature effect is observed (Figures $\mathrm{S} 6 \mathrm{~b}$ and S8). Both compounds exhibit a large Stokes shift (ca. $1.7 \mathrm{eV}$ ) for the dominant triplet emission. 

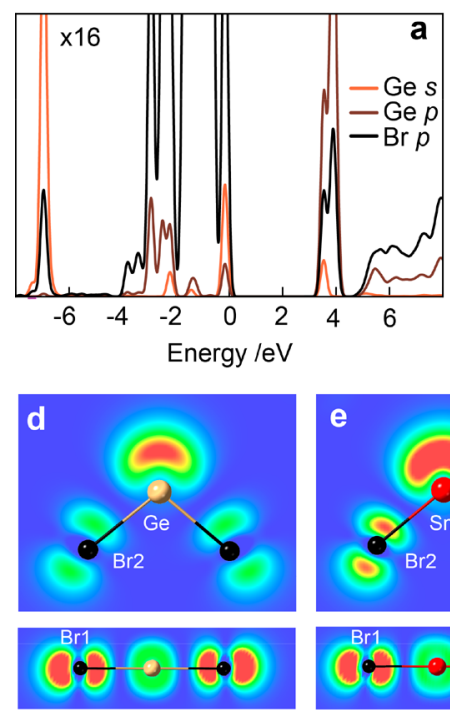
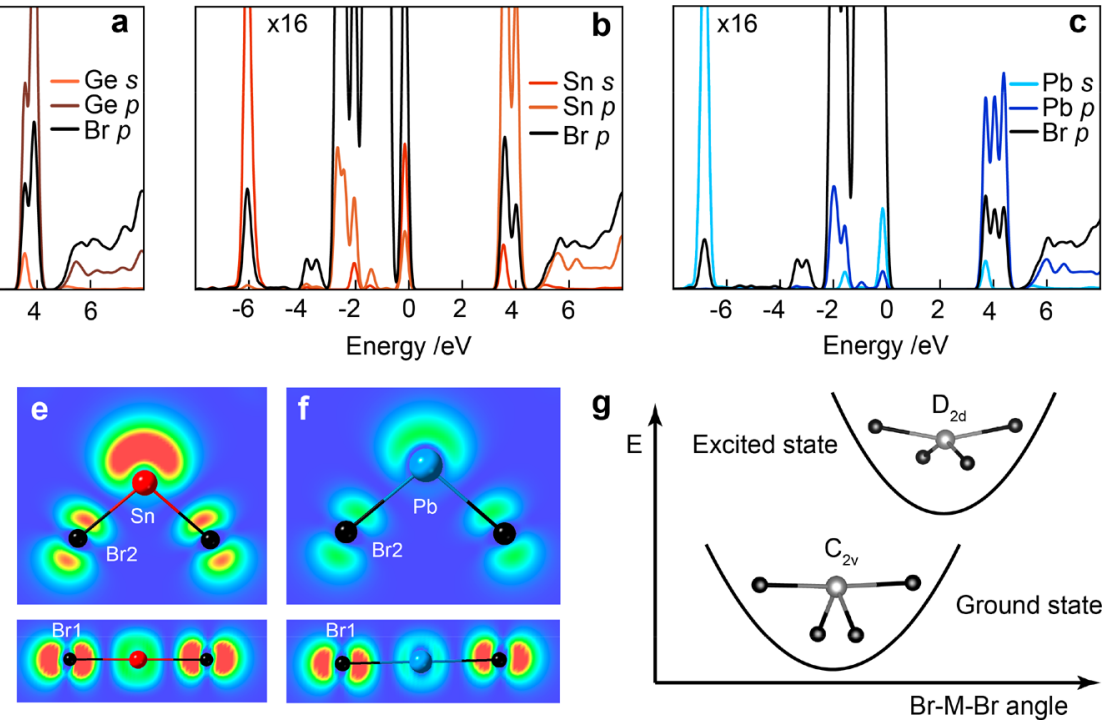

Figure 3. $(\mathrm{a}-\mathrm{c})$ Atomic-orbital-projected density of states for $\mathrm{M} \mathrm{s}, \mathrm{M}$ p, and $\mathrm{Br} \mathrm{p}$ contributions in $\mathrm{Bmpip}_{2} \mathrm{MBr}_{4}(\mathrm{M}=\mathrm{Ge}, \mathrm{Sn}, \mathrm{Pb})$. $(\mathrm{d}-\mathrm{f})$ Respective electron density maps at the top of the valence band (ground state), plotted between 0 and $0-0.011$ e/ $\AA^{3}$. (g) Schematics for the ground-state and excited-state geometries.

In the analogous $\mathrm{Pb}$ compound, the Stokes shift is almost two times smaller (ca. $0.9 \mathrm{eV}$ ), and there is one pronounced emission band at RT, centered at $470 \mathrm{~nm}$. The emission decay function consists of two very distinct components: $\tau=230 \mathrm{ps}$ and $\tau=66 \mathrm{~ns}$ (Figure S5). The faster emission becomes dominant upon cooling from 300 to $10 \mathrm{~K}$ (Figure S9). In addition, the streak-camera captures the emergence of a fast, sub-100 ps singlet emission at $435 \mathrm{~nm}$, coexisting with the 470 $\mathrm{nm}$ peak (Figure S10). The optical properties of all three compounds can be explained on the basis of the $\mathrm{ns}^{2}$ free ion energy levels (Figure 2d). For Ge and Sn, the Jahn-Teller (JT) perturbation of the ground state contributes the most to the splitting of the energy levels. For the $\mathrm{Pb}$ ion, however, spin-orbit coupling (SOC) perturbation is much stronger than $\mathrm{JT}$; this interplay is well-known for $\mathrm{ns}^{2}$-metal centered emission. ${ }^{13}$ This results in a smaller Stokes shift as well as more efficient mixing of ${ }^{1} \mathrm{P}$ and ${ }^{3} \mathrm{P}$ states for a heavier metal $(\mathrm{Pb}, \mathrm{Bi})$. In addition, the slower component of the emission at $470 \mathrm{~nm}$ ( $\tau=66 \mathrm{~ns})$ in $\mathrm{Bmpip}_{2} \mathrm{PbBr}_{4}$ can be attributed to the ${ }^{3} \mathrm{P}_{0} \rightarrow{ }^{1} \mathrm{~S}_{0}$ transition, which becomes allowed only for $\mathrm{Pb}$ due to singlet/ triplet mixing.

We have investigated electronic structures of the synthesized compounds on the density functional theory (DFT) level utilizing the Perdew-Burke-Ernzerhof (PBE) functional as implemented in the Vienna $a b$ initio simulation package (VASP), initially without including SOC (Figure 3). When SOC and hybrid DFT functionals have been added, the qualitative picture remains the same (Figures S11 and S12). The electronic structure of $\mathrm{Bmpip}_{2} \mathrm{MBr}_{4}$ complexes is highly localized on $\mathrm{MBr}_{4}$ fragments, seen as flat electronic bands (Figure S13). The electronic density of states corroborates that the stereochemical activity of the lone pair depends not only on the metal but also on the halide. This dependence on both metal and anion has been previously theoretically investigated in monoxides and monochalcogenides of $\mathrm{Ge}, \mathrm{Sn}$, and $\mathrm{Pb}{ }^{14}$ Upon examining the projected density of states (PDOS, Figure 3 ), the main contribution of the metal s-states can be found below $-5 \mathrm{eV}$. The contribution at the top of the valence band, around $0 \mathrm{eV}$, is smaller. This contribution determines the stereoactivity of the lone pair. In the case of Sn, s-states near the Fermi level are broader and better mixed with $\mathrm{Br}$ p-states. The mixing is additionally mediated by empty $\mathrm{M}$ p-states. This corroborates the assumption that the lone pair is more active in the case of $\mathrm{Sn}$. The lone pair stereoactivity manifests itself also through the repulsion with the axial halide atoms, adjusting the bond angles (Tables S6-S13).

In the excited state, the sp-mixing of the HOMO no longer stabilizes the lone pair: the energy gain of HOMO stabilization is compensated by the energy of the electron promoted to the LUMO. For similar $\mathrm{Sb}$ (III) chloride complexes in acetonitrile, Nikol et al. have argued that the structure, therefore, relaxes toward $T_{d}$ symmetry upon excitation. ${ }^{15}$ The energy loss associated with this change is reflected in the Stokes shift. We have calculated the excited-state geometry using the PBE functional and unrestricted Kohn-Sham approach with triplet multiplicity as implemented in the cp $2 \mathrm{k}$ code. The results suggest that in the excited state the structure changes from $C_{2 v}$ symmetry to another lower symmetry derivative of $T_{d}$, e.g., $D_{2 d}$ (Figure $3 \mathrm{~g}$ ). In this symmetry, the gain in energy from the promotion of an electron from the HOMO to LUMO overcomes the HOMO stabilization in the $C_{2 v}$ geometry.

The scope of applications for luminescent zero-dimensional metal halides has thus far been limited to solid-state lighting, exploiting their broadband emission. ${ }^{7 b, 16}$ Herein, we outline yet another perspective application-X-ray detection by scintillation-that harnesses specific advantages of these compounds; namely, high X-ray absorptivity by heavy elements, highly localized emission centers, and large emission Stokes shifts for efficient light out-coupling. As a showcase example, blue-emissive $\mathrm{Bmpip}_{2} \mathrm{PbBr}_{4}$ and red-emissive $\mathrm{Bmpip}_{2} \mathrm{SnBr}_{4}$ has been compared with the typical commercial $\mathrm{NaI}$ :Tl scintillator. In such an X-ray excited PL comparative experiment, about one half of a gram of $\mathrm{Bmpip}_{2} \mathrm{PbBr}_{4}$ and Bmpip $_{2} \mathrm{SnBr}_{4}$ was pressed into pellets $(13 \mathrm{~mm} \times 2.5 \mathrm{~mm}$, see the Supporting Information) and illuminated with a $50 \mathrm{kV} \mathrm{Ag}$ $\mathrm{X}$-ray tube. The thickness of such pellets is estimated to ensure nearly complete absorption of X-rays, based on calculated Xray absorptivities of these materials (Figure S14). X-ray 
transmittance images (Figure S15) convey that the pellets do absorb more than $90 \%$ of the incident X-ray photons. The radioluminescence spectra of $\mathrm{NaI}: \mathrm{Tl}, \mathrm{Bmpip}_{2} \mathrm{PbBr}_{4}$, and $\mathrm{Bmpip}_{2} \mathrm{SnBr}_{4}$ excited by a $50 \mathrm{kV} \mathrm{Ag}$ tube and corrected for background and spectral sensitivity of the system exhibit similar intensities (Figure 4a,b). A visual comparison of the

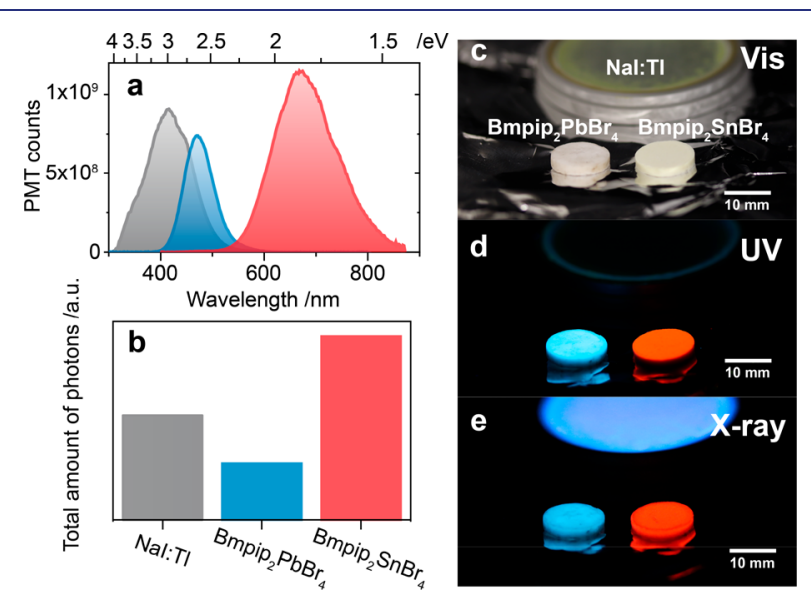

Figure 4. (a) X-ray fluorescence spectra under $50 \mathrm{kV} \mathrm{Ag}$ tube irradiation. (b) The normalized total amount of photons comparison with the same X-ray excitation source. NaI:Tl commercial scintillator, $\mathrm{Bmpip}_{2} \mathrm{PbBr}_{4}$ pellet, and $\mathrm{Bmpip}_{2} \mathrm{SnBr}_{4}$ pellet under (c) ambient light, (d) UV irradiation $(365 \mathrm{~nm})$, and (e) X-ray irradiation with a Rayme DG-073B-DC X-ray tube as an excitation source $(70 \mathrm{kV}, 1 \mathrm{~mA}, 1.8 \mathrm{~s}$ exposure time).

studied materials under daylight, UV light, and X-rays is presented in Figure $4 c-$ e. The noteworthy performance of Bmpip $\mathrm{MBr}_{4}$ compounds warrants further detailed investigations. Future practical avenues include the engineering of the optically homogeneous medium, such as sufficiently large single-crystals or fine dispersions in polymers. These forms of low-dimensional metal halides will allow for accurate measurements of the radioluminescence quantum yields.

In conclusion, $\mathrm{Bmpip}_{2} \mathrm{MBr}_{4}(\mathrm{M}=\mathrm{Ge}, \mathrm{Sn}, \mathrm{Pb})$ compounds feature unusual disphenoidal coordination. $\mathrm{Ge}$ and $\mathrm{Sn}$ compounds display both singlet and triplet exciton emission. The computational study emphasized the contribution of the lone pair. In the practical context, $\mathrm{Pb}$ and $\mathrm{Sn}$ compounds have been shown to be potent X-ray fluorophores, comparable to NaI:Tl. Future work should focus on the systematic exploration of such metal halides as scintillators for hard radiation detection.

\section{ASSOCIATED CONTENT}

\section{S Supporting Information}

The Supporting Information is available free of charge on the ACS Publications website at DOI: 10.1021/jacs.9b02365.

Crystallographic data for $\mathrm{C}_{20} \mathrm{H}_{44} \mathrm{~N}_{2} \mathrm{GeBr}_{4}$ (CIF)

Crystallographic data for $\mathrm{C}_{20} \mathrm{H}_{44} \mathrm{~N}_{2} \mathrm{PbBr}_{4}$ (CIF)

Crystallographic data for $\mathrm{C}_{20} \mathrm{H}_{44} \mathrm{SnBr}_{4}$ (CIF)

Methods and additional figures including XRD patterns, photoluminescence measurement spectra, 2D PL vs PLE map, luminescence decay, temperature-dependent emission decay curves, time-resolved emission, energy level diagram, total energy comparison, DOS comparison, $\mathrm{X}$ ray absorbance vs photon energy, and transmission image (PDF)

\section{AUTHOR INFORMATION}

\section{Corresponding Author}

*mvkovalenko@ethz.ch

ORCID $\odot$

Yevhen Shynkarenko: 0000-0002-1587-1752

Sergii Yakunin: 0000-0002-6409-0565

Alexandra Brumberg: 0000-0003-2512-4686

Richard D. Schaller: 0000-0001-9696-8830

Maksym V. Kovalenko: 0000-0002-6396-8938

Notes

The authors declare no competing financial interest.

\section{ACKNOWLEDGMENTS}

The authors are indebted to Dr. M. Wörle for assistance with structure determination and Dr. S. Ochsenbein for helpful discussions. This work was performed, in part, at the Center for Nanoscale Materials, a U.S. Department of Energy Office of Science User Facility, and supported by the U.S. Department of Energy, Office of Science, under Contract DE-AC0206CH11357. A.B. acknowledges support from the National Science Foundation Graduate Research Fellowship Program under Grant DGE-1324585.

\section{REFERENCES}

(1) (a) Stranks, S. D.; Snaith, H. J. Metal-Halide Perovskites for Photovoltaic and Light-Emitting Devices. Nat. Nanotechnol. 2015, 10 (5), 391-402. (b) Luo, J. J.; Wang, X. M.; Li, S. R.; Liu, J.; Guo, Y. M.; Niu, G. D.; Yao, L.; Fu, Y. H.; Gao, L.; Dong, Q. S.; Zhao, C. Y.; Leng, M. Y.; Ma, F. S.; Liang, W. X.; Wang, L. D.; Jin, S. Y.; Han, J. B.; Zhang, L. J.; Etheridge, J.; Wang, J. B.; Yan, Y. F.; Sargent, E. H.; Tang, J. Efficient and stable emission of warm-white light from leadfree halide double perovskites. Nature 2018, 563 (7732), 541-545.

(2) Utzat, H.; Sun, W.; Kaplan, A. E. K.; Krieg, F.; Ginterseder, M.; Spokoyny, B.; Klein, N. D.; Shulenberger, K. E.; Perkinson, C. F.; Kovalenko, M. V.; Bawendi, M. G. Coherent single-photon emission from colloidal lead halide perovskite quantum dots. Science 2019, 363 (6431), 1068-1072.

(3) (a) Burschka, J.; Pellet, N.; Moon, S. J.; Humphry-Baker, R.; Gao, P.; Nazeeruddin, M. K.; Gratzel, M. Sequential Deposition as a Route to High-Performance Perovskite-Sensitized Solar Cells. Nature 2013, 499 (7458), 316-320. (b) Lee, M. M.; Teuscher, J.; Miyasaka, T.; Murakami, T. N.; Snaith, H. J. Efficient Hybrid Solar Cells Based on Meso-Superstructured Organometal Halide Perovskites. Science 2012, 338 (6107), 643-647. (c) Yakunin, S.; Dirin, D. N.; Shynkarenko, Y.; Morad, V.; Cherniukh, I.; Nazarenko, O.; Kreil, D.; Nauser, T.; Kovalenko, M. V. Detection of Gamma Photons Using Solution-Grown Single Crystals of Hybrid Lead Halide Perovskites. Nat. Photonics 2016, 10 (9), 585-589. (d) Stoumpos, C. C.; Malliakas, C. D.; Peters, J. A.; Liu, Z. F.; Sebastian, M.; Im, J.; Chasapis, T. C.; Wibowo, A. C.; Chung, D. Y.; Freeman, A. J.; Wessels, B. W.; Kanatzidis, M. G. Crystal Growth of the Perovskite Semiconductor $\mathrm{CsPBBr}_{3}$ : A New Material for High-Energy Radiation Detection. Cryst. Growth Des. 2013, 13 (7), 2722-2727. (e) Dou, L. T.; Yang, Y.; You, J. B.; Hong, Z. R.; Chang, W. H.; Li, G.; Yang, Y. Solution-Processed Hybrid Perovskite Photodetectors With High Detectivity. Nat. Commun. 2014, 5, 5404-5410. (f) Hu, X.; Zhang, X. D.; Liang, L.; Bao, J.; Li, S.; Yang, W. L.; Xie, Y. High-Performance Flexible Broadband Photodetector Based on Organolead Halide Perovskite. Adv. Funct. Mater. 2014, 24 (46), 7373-7380. (g) Yakunin, S.; Shynkarenko, Y.; Dirin, D. N.; Cherniukh, I.; Kovalenko, M. V. Non-Dissipative Internal Optical Filtering with Solution-Grown Perovskite Single Crystals for Full-Colour Imaging. NPG Asia Mater. 2017, 9, e431.

(4) (a) Schmidt, L. C.; Pertegas, A.; Gonzalez-Carrero, S.; Malinkiewicz, O.; Agouram, S.; Espallargas, G. M.; Bolink, H. J.; 
Galian, R. E.; Perez-Prieto, J. Nontemplate Synthesis of $\mathrm{CH}_{3} \mathrm{NH}_{3} \mathrm{PbBr}_{3}$ Perovskite Nanoparticles. J. Am. Chem. Soc. 2014, 136 (3), 850-853. (b) Protesescu, L.; Yakunin, S.; Bodnarchuk, M. I.; Krieg, F.; Caputo, R.; Hendon, C. H.; Yang, R. X.; Walsh, A.; Kovalenko, M. V. Nanocrystals of Cesium Lead Halide Perovskites $\left(\mathrm{CsPbX}_{3}, \mathrm{X}=\mathrm{Cl}, \mathrm{Br}\right.$, and I): Novel Optoelectronic Materials Showing Bright Emission with Wide Color Gamut. Nano Lett. 2015, 15 (6), 3692-3696. (c) Shamsi, J.; Urban, A. S.; Imran, M.; De Trizio, L.; Manna, L. Metal Halide Perovskite Nanocrystals: Synthesis, PostSynthesis Modifications, and Their Optical Properties. Chem. Rev. 2019, 119, 3296-3348.

(5) (a) Mao, L. L.; Guo, P. J.; Kepenekian, M.; Hadar, I.; Katan, C.; Even, J.; Schaller, R. D.; Stoumpos, C. C.; Kanatzidis, M. G. Structural Diversity in White-Light-Emitting Hybrid Lead Bromide Perovskites. J. Am. Chem. Soc. 2018, 140 (40), 13078-13088. (b) Lin, H. R.; Zhou, C. K.; Tian, Y.; Siegrist, T.; Ma, B. W. Low-Dimensional Organometal Halide Perovskites. ACS Energy Lett. 2018, 3 (1), 5462.

(6) (a) Fu, P.; Huang, M.; Shang, Y.; Yu, N.; Zhou, H.-L.; Zhang, Y.B.; Chen, S.; Gong, J.; Ning, Z. Hollow Two-Dimensional Tin Bromide Perovskite with Tunable Broadband Emission. ACS Appl. Mater. Interfaces 2018, 10 (40), 34363-34369. (b) Dohner, E. R.; Jaffe, A.; Bradshaw, L. R.; Karunadasa, H. I. Intrinsic White-Light Emission from Layered Hybrid Perovskites. J. Am. Chem. Soc. 2014, 136 (38), 13154-13157. (c) Smith, M. D.; Karunadasa, H. I. WhiteLight Emission from Layered Halide Perovskites. Acc. Chem. Res. 2018, 51 (3), 619-627. (d) Dohner, E. R.; Hoke, E. T.; Karunadasa, H. I. Self-Assembly of Broadband White-Light Emitters. J. Am. Chem. Soc. 2014, 136 (5), 1718-1721.

(7) (a) Benin, B. M.; Dirin, D. N.; Morad, V.; Worle, M.; Yakunin, S.; Raino, G.; Nazarenko, O.; Fischer, M.; Infante, I.; Kovalenko, M. V. Highly Emissive Self-Trapped Excitons in Fully Inorganic ZeroDimensional Tin Halides. Angew. Chem., Int. Ed. 2018, 57 (35), 11329-11333. (b) Zhou, C. K.; Lin, H. R.; Tian, Y.; Yuan, Z.; Clark, R.; Chen, B. H.; van de Burgt, L. J.; Wang, J. C.; Zhou, Y.; Hanson, K.; Meisner, Q. J.; Neu, J.; Besara, T.; Siegrist, T.; Lambers, E.; Djurovich, P.; Ma, B. W. Luminescent zero-dimensional organic metal halide hybrids with near-unity quantum efficiency. Chem. Sci. 2018, 9 (3), 586-593. (c) Zhou, J.; Li, M. Z.; Ning, L. X.; Zhang, R. L.; Molokeev, M. S.; Zhao, J.; Yang, S. Q.; Han, K. L.; Xia, Z. G. BroadBand Emission in a Zero-Dimensional Hybrid Organic [PbBr6] Trimer with Intrinsic Vacancies. J. Phys. Chem. Lett. 2019, 10 (6), 1337-1341. (d) Roccanova, R.; Yangui, A.; Nhalil, H.; Shi, H.; Du, M.-H.; Saparov, B. Near-Unity Photoluminescence Quantum Yield in Blue-Emitting Cs3Cu2Br5-xIx $(0 \leq x \leq 5)$. ACS Applied Electronic Materials 2019, 1 (3), 269-274.

(8) Vogler, A.; Nikol, H. The Structures of $s^{2}$ Metal Complexes in the Ground and $s p$ Excited States. Comments Inorg. Chem. 1993, 14 (4), 245-261.

(9) (a) Degruijter, W. C. Luminescence of Lead Chloride and Lead Bromide Single Crystals. Phys. Lett. A 1971, 34 (5), 251-252. (b) Iwanaga, M.; Watanabe, M.; Hayashi, T. Charge Separation of Excitons and the Radiative Recombination Process in $\mathrm{PbBr}_{2}$ Crystals. Phys. Rev. B: Condens. Matter Mater. Phys. 2000, 62 (16), 1076610773. (c) Iwanaga, M.; Shirai, M.; Tanaka, K.; Hayashi, T. SelfTrapped States and Related Luminescence in $\mathrm{PbCl}_{2}$ Crystals. Phys. Rev. B: Condens. Matter Mater. Phys. 2002, 66 (6), 4304-4312. (d) Nara, J.; Adachi, S. Optical Properties of $\mathrm{SnCl}_{2}$ Phosphor. J. Appl. Phys. 2011, 109 (8), 3539-3546. (e) Eijkelenkamp, A. J. H. Photoluminescence of $\mathrm{PbBr}_{2}, \mathrm{PbCl}_{2}$ and Beta- $\mathrm{PbF}_{2}$ Single-Crystals. J. Lumin. 1977, 15 (2), 217-225. (f) Liidja, G.; Plekhanov, V. LowTemperature X-ray and Photoluminescence of Lead Halide Crystals. J. Lumin. 1973, 6 (2), 71-76.

(10) Kang, J.; Wang, L. W. High Defect Tolerance in Lead Halide Perovskite $\mathrm{CsPbBr}_{3}$. J. Phys. Chem. Lett. 2017, 8 (2), 489-493.

(11) (a) Blachnik, R.; Reuter, H.; Wiest, T.; Eickmeier, H. Crystal Structure of 1,2-Vinylenbis(triphenylphosphonium) Dibromide Dihydrate, $\left[\mathrm{Ph}_{3} \mathrm{P}-\mathrm{CH}=\mathrm{CH}-\mathrm{PPh}_{3}\right][\mathrm{Br}]_{2} \cdot 2 \mathrm{H}_{2} \mathrm{O}$. Z. Kristallogr. - New Cryst. Struct. 2000, 215 (2), 251-252. (b) Gröger, H.; Lode, C.; Vollmer,
H.; Krautscheid, H.; Lebedkin, S. Bromoplumbate mit Kettenförmigen und Isolierten Anionen: $\left(\mathrm{Bzl}_{4} \mathrm{P}\right)_{2}\left[\mathrm{~Pb}_{3} \mathrm{Br}_{8}\right]$, $\left(\mathrm{Bzl}_{4} \mathrm{P}\right)_{2}\left[\mathrm{~Pb}_{3} \mathrm{Br}_{8}(\mathrm{dmf})_{2}\right], \quad\left(\mathrm{Bzl}_{4} \mathrm{P}\right)\left[\mathrm{PbBr}_{3}\right],\left(\mathrm{Bzl}_{4} \mathrm{P}\right)_{2}\left[\mathrm{PbBr}_{4}\right]$ und $\left(\mathrm{Bzl}_{4} \mathrm{P}\right)_{4}\left[\mathrm{~Pb}_{2} \mathrm{Br}_{6}\right]\left[\mathrm{PbBr}_{4}\right]$. Z. Anorg. Allg. Chem. 2002, 628 (1), 57-62.

(12) Li, W.; Zhou, L. J.; Prezhdo, O. V.; Akimov, A. V. Spin-Orbit Interactions Greatly Accelerate Nonradiative Dynamics in Lead Halide Perovskites. ACS Energy Lett. 2018, 3 (9), 2159-2166.

(13) Jacobs, P. W. M. Alkali-Halide Crystals Containing Impurity Ions with the $\mathrm{ns}^{2}$ Ground-State Electronic Configuration. J. Phys. Chem. Solids 1991, 52 (1), 35-67.

(14) (a) Walsh, A.; Watson, G. W. Influence of the Anion on Lone Pair Formation in Sn(II) Monochalcogenides: A DFT Study. J. Phys. Chem. B 2005, 109 (40), 18868-18875. (b) Walsh, A.; Watson, G. $\mathrm{W}$. The Origin of the Stereochemically Active $\mathrm{Pb}(\mathrm{II})$ Lone Pair: DFT Calculations on $\mathrm{PbO}$ and PbS. J. Solid State Chem. 2005, 178 (5), $1422-1428$.

(15) Nikol, H.; Becht, A.; Vogler, A. Photoluminescence of Germanium(II), Tin(II), and Lead(II) Chloride Complexes in Solution. Inorg. Chem. 1992, 31 (15), 3277-3279.

(16) Worku, M.; Tian, Y.; Zhou, C. K.; Lee, S.; Meisner, Q.; Zhou, Y.; Ma, B. W. Sunlike White-Light-Emitting Diodes Based on ZeroDimensional Organic Metal Halide Hybrids. ACS Appl. Mater. Interfaces 2018, 10 (36), 30051-30057. 